\title{
Defective Beta Adrenergic Response of Cystic Fibrosis Sweat Glands In Vivo and In Vitro
}

Kenzo Sato and Fusako Sato

Marshall Dermatology Research Laboratories, Department of Dermatology, University of Iowa Hospitals and Clinics, Iowa City, Iowa 52242

bstract. Abnormal ductal $\mathrm{NaCl}$ absorption has been known as the only defect in cystic fibrosis (CF) sweat glands. We have fortuitously found that the secretory portion of CF sweat glands is also abnormal in that it failed to show a sweating response to beta adrenergic stimulation (isoproterenol, [ISO]) both in vivo and in vitro. For the in vitro sweat test, eccrine sweat glands were isolated from skin biopsy specimens of the forearm, cannulated, and stimulated to secrete sweat. All 14 isolated CF sweat glands failed to respond to ISO + theophylline (TH, as aminophylline), but 17 of 18 control glands responded with a mean rate (SR) of $1.1 \mathrm{nl} / \mathrm{min}$ per gland. Cholinergic responsiveness of isolated CF sweat glands was comparable with that of control glands. The in vivo sweat test was performed by intradermal injection in the forearm of $0.2 \mathrm{ml}$ of 2.4 or $8 \times 10^{-5} \mathrm{M}$ ISO with or without $10^{-2} \mathrm{M} \mathrm{TH}$ (and $1.4 \times 10^{-4} \mathrm{M}$ atropine as a necessary anticholinergic agent). The beads of sweat secreted into the oil-filled sweat collection ring glued to the skin were then collected with a glass capillary under a stereomicroscope. Of $28 \mathrm{CF}$ patients, 26 failed to show a secretory response to intradermal injection of ISO $+\mathrm{TH}$, and 2 CF patients gave SR of $<0.007 \mathrm{nl} / \mathrm{min}$ per gland in the first test but no response in the repeat test performed later. In contrast, all 35 age- and sex-matched control subjects responded with the mean SR of $0.72 \mathrm{nl} / \mathrm{min}$ per gland. Response of CF patients to epinephrine and phenylephrine was comparable with control, indicating that the alpha adrenergic responsiveness of CF sweat glands is not defective. A preliminary attempt was made to determine tissue cyclic AMP accumulation by radioim-

Received for publication 9 August 1984 and in revised form 23 February 1984.

J. Clin. Invest.

(C) The American Society for Clinical Investigation, Inc. 0021-9738/84/06/1763/09 \$1.00

Volume 73, June 1984, 1763-1771 munoassay in isolated sweat glands. No significant difference was observed between CF and control glands in their maximal accumulation of tissue cAMP in response to ISO or ISO $+\mathrm{TH}$, except that the rise time of ISO + TH-induced cAMP accumulation in CF glands was significantly slower during the first $5 \mathrm{~min}$ of incubation. The data suggest that beta adrenergic regulation is abnormal in CF sweat glands and justifies further investigations into the mechanism of beta adrenergic regulation of the eccrine sweat gland in both normal and CF subjects.

\section{Introduction}

The eccrine sweat gland has long attracted the attention of cystic fibrosis $(\mathrm{CF})^{1}$ researchers because the elevated sweat $\mathrm{NaCl}$ concentration is the cornerstone of the diagnosis (1). It appears to be firmly established that the elevated sweat $\mathrm{NaCl}$ is due to impairment of the $\mathrm{CF}$ sweat duct to reabsorb $\mathrm{NaCl}$ from apparently normal primary sweat formed in the secretory portion $(2,3,4)$. In contrast, the function of the secretory portion itself has been assumed to be normal in CF. Recent data from the authors' laboratory indicates that the isolated cannulated monkey palm eccrine sweat gland is controlled by alpha and beta adrenergic as well as cholinergic stimulation (5). Further, the role of $\mathrm{Ca}$ in the intracellular processes during cholinergic sweating and that of intracellular cyclic AMP (cAMP) in the beta adrenergic mechanism of sweating have been suggested $(6,7)$. During extension of a similar pharmacological approach to isolated human sweat glands, we fortuitously discovered that isolated, cannulated CF sweat glands failed to secrete sweat in response to isoproterenol (ISO) while their cholinergic responsiveness was apparently normal. We report herein that this defective responsiveness of the $C F$ sweat gland was present in vivo as well as in vitro, although preliminary data indicate that the isolated CF sweat glands accumulated intracellular cAMP apparently normally in response to ISO.

1. Abbreviations used in this paper: AT, atropine; CF, cystic fibrosis; $\mathrm{EP}$, epinephrine; ISO, isoproterenol; KRB, Krebs-Ringer bicarbonate; $\mathrm{MCH}$, methacholine; PL, phenylephrine; PR, propranol; TH, theophylline. 


\section{Methods}

Selection of CF patients (homozygotes). 35 CF patients, $10 \mathrm{yr}$ of age or older, were recruited from a list of about 150 patients registered at the Cystic Fibrosis Center, University of Iowa Hospitals and Clinics. The diagnosis of $\mathrm{CF}$ was made based on a positive sweat test (chloride concentration of $>60 \mathrm{mM}$ in $50 \mathrm{mg}$ or larger sweat samples obtained by routine diagnostic pilocarpine iontophoresis) and a combination of pulmonary, gastrointestinal, and pancreatic disorders usually associated with CF. Skin biopsy was limited to male patients, 16 to $28 \mathrm{yr}$ of age. Some CF patients allowed us to take two skin biopsies. None of the 48 control subjects (all Caucasian) used for in vivo skin test or for skin biopsy had taken antibiotics or adrenergic agonists for 2 mo prior to the sweat test or skin biopsy. Among the $35 \mathrm{CF}$ patients who participated in the in vivo sweat test, 10 were taking pancreatic enzymes; 10 had taken various antibiotics (Amoxicillin, Tetracycline, Bactrim, Dicloxacillin, Ampicillin, Vibramycin, Tobramycin, Gentamicin); 2, terbutaline; 2 , isoprenaline; and 3 , theophylline intermittently during the preceding 2 mo. All CF subjects used for skin biopsy were free of adrenergic agonists or theophylline (TH) for at least $1 \mathrm{yr}$ prior to the biopsy. All the patients were in no acute distress and physically active at the time of the sweat test.

Skin biopsy and sweat gland isolation. After local block anesthesia using $1 \%$ lidocaine without epinephrine (EP), elliptical pieces of skin,
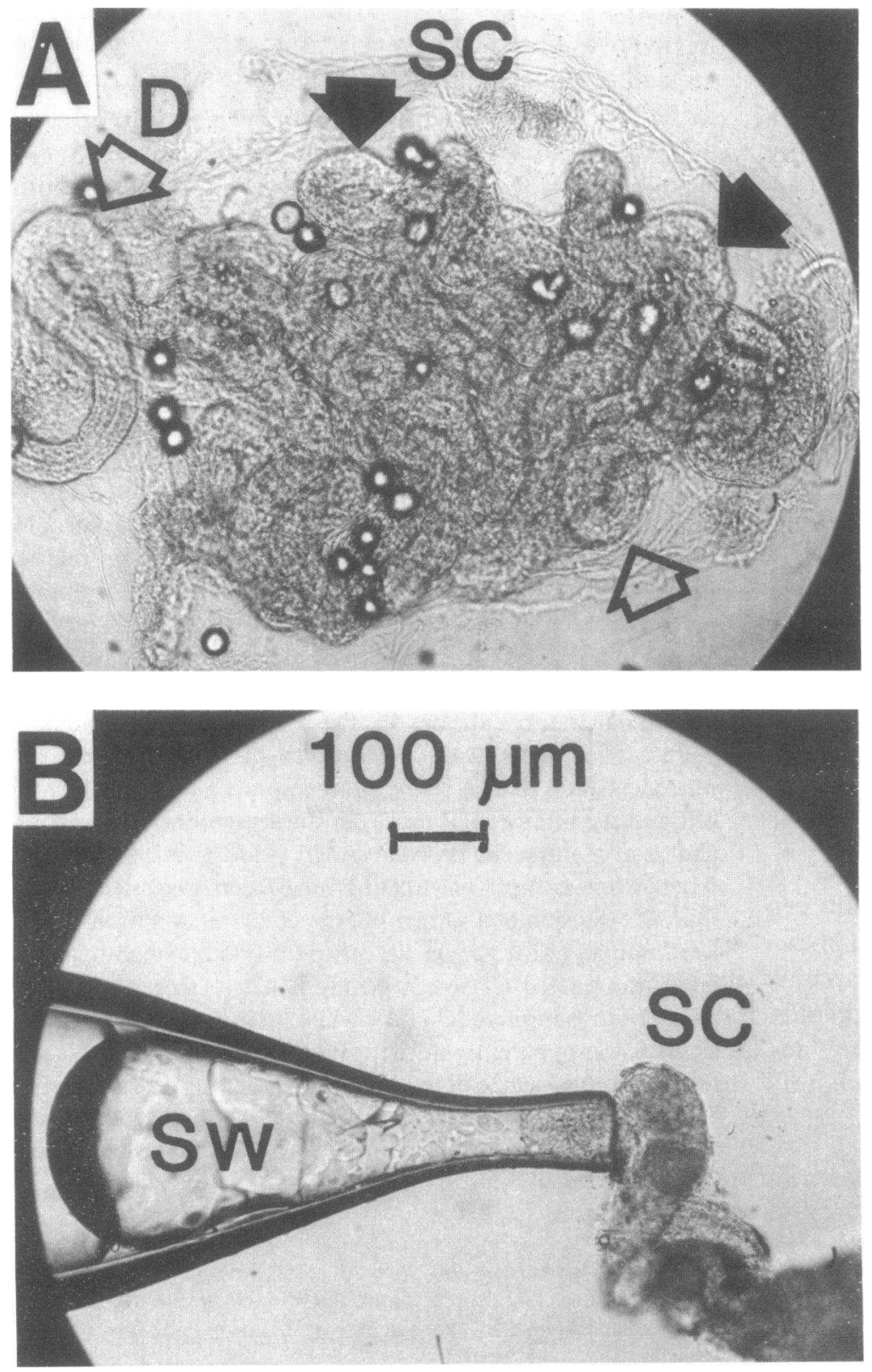

Figure 1. An isolated sweat gland from a patient with $C F$. $(A)$ Isolated whole sweat gland (the duct $[\mathrm{D}$, indicated by empty arrows] and the secretory coil [SC, solid arrows]). (B) An isolated secretory portion of the CF sweat gland (SC) held in a constriction pipette; SW, sweat secreted in an oil-filled pipet. 
$1 \mathrm{~cm}$ long and $0.5 \mathrm{~cm}$ wide, were excised from the forearms of 12 physically active adult $\mathrm{CF}$ male patients and 14 age-matched equally physically active control males, and the surgical wound was primarily closed with a running subcuticular suture. The direct infusion of lidocaine into the excised skin was avoided. The biopsy skin was blotted of blood, sliced freehand with a razor blade, and washed in several changes of chilled Krebs-Ringer bicarbonate (KRB) solution containing (in $\mathrm{mM}$ ): $115 \mathrm{NaCl}, 10 \mathrm{Na}$-acetate, $5 \mathrm{KCl}, 1.2 \mathrm{MgCl}_{2}, 25 \mathrm{NaHCO}_{3}, 1.2 \mathrm{NaH}_{2} \mathrm{PO}_{4}$, 5 glucose, and $10 \mathrm{mg} / 100 \mathrm{ml}$ fatty acid-free human serum albumin (Sigma Chemical Co., St. Louis, MO). The pH of this medium was 7.45 when equilibrated with $5 \% \mathrm{CO}_{2}: 95 \% \mathrm{O}_{2}$. Single sweat glands were isolated at $10^{\circ} \mathrm{C}$ under a stereomicroscope by gently teasing away periglandular collagen fibers with sharp forceps (5) (Fig. $1 A$ ).

Sweat induction in vitro. The open end of the duct near the junction of the proximal and the distal duct was held by suction in a siliconized constriction pipette as described previously (5). In some sweat glands, sweat was collected directly from the end of the secretory coil held in a pipette, as shown in Fig. $1 B$. Because the ductal reabsorption of water, if any, is not significantly large (5), the ductal sweat was conveniently regarded as the same as true secretory rate and was treated together with the sweat rate obtained directly from the secretory coil. The tissueglass junction inside the constriction pipette was sealed with Sylgard 184 (Dow Corning Corp., Midland, MI), and the pipette was filled with water-saturated paraffin oil. The temperature of the bath (KRB) was then raised from 10 to $37^{\circ} \mathrm{C}$. Sweat secretion was then initiated by various concentrations of methacholine $(\mathrm{MCH})$. Sweat glands which failed to respond to $\mathrm{MCH}$ were discarded because they were most likely damaged during isolation or occluded with tissue debris. In such glands, the proximal tubular lumen was often dilated and/or tiny particles were seen flowing through the lumen proximal to the possible site of damage. Likewise, sweat glands that failed to produce $>80 \%$ of the control (first) $\mathrm{MCH}$ sweat rate upon restimulation (e.g., $\mathrm{MCH}$ at $70 \mathrm{~min}$ in Fig. 5) were excluded from the study. About $50 \%$ of the initially responding glands did not meet this last criterion. The number of sweat glands successfully isolated for the sweat induction study from each skin biopsy varied from zero (in two CF patients) to three (in one CF patient) glands. The data from all sweat glands which met the criteria set forth above were treated together.

Following initial stimulation with $\mathrm{MCH}$, the incubation bath was replaced with several changes of fresh KRB containing $10^{-7} \mathrm{M}$ atropine (AT), which immediately stopped $\mathrm{MCH}$ sweating. ISO or EP was then added to the bath at different concentrations with or without $10^{-3} \mathrm{M}$ (or in some glands $10^{-2} \mathrm{M}$ ) TH. Collection of sweat in an oil-filled sampling pipette and calibration of sweat volume are essentially the same as described previously (5).

Intradermal sweat test in vivo. The anaerobic method of sweat collection (8) was used with minor modifications. Briefly, a teflon ring (sweat collection chamber) with a 7-mm hole in the center was glued to the skin of the forearm using a contact cement (Fig. 2). Paraffin oil saturated with water was poured into the trough of the chamber. At a room temperature of $24^{\circ} \mathrm{C}$, no sweat secretion was detected when observed under a stereomicroscope for as long as $1 \mathrm{~h}$. At time zero, a 30gauge needle was inserted into the dermis at the center of the test site and $0.2 \mathrm{ml}$ of injection solution was infiltrated so as to cover the test area of $0.38 \mathrm{~cm}^{2}$ with a uniform blanching (injection) wheal (Fig. 2). This is of critical importance with ISO injection because deep or subcutaneous injection can easily produce a false negative sweating response. To insure obtaining the maximal sweat rate, we always performed intradermal injection of adrenergic drugs in duplicate in both control and CF subjects, and employed the higher sweat rate and discarded the other.
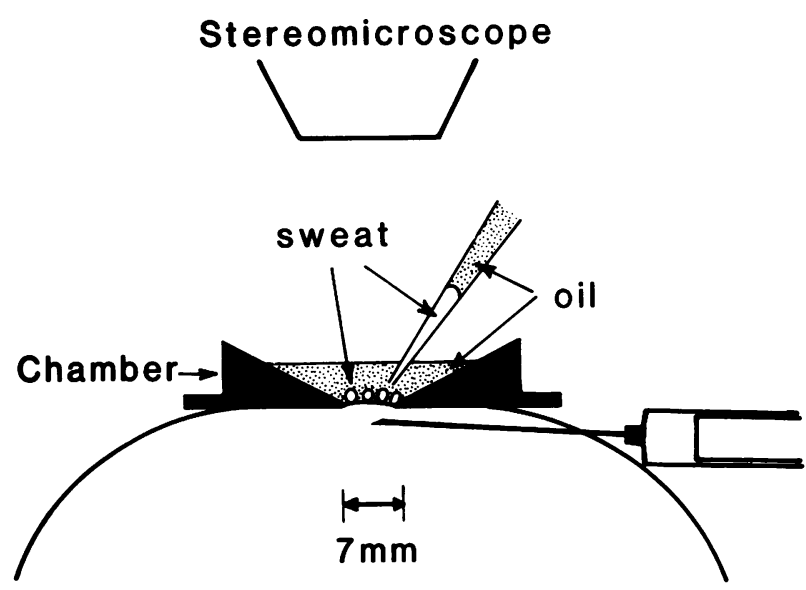

\section{Forearm}

Figure 2. A schematic representation of a modified anaerobic sweat collection used for in vivo sweating studies. Beads of sweat secreted into oil were collected with an oil-filled glass capillary.

In other words, a negative response of a CF patient to ISO + TH was always confirmed in duplicate. After injection, beads of sweat secreted into oil were collected with an oil-filled glass pipette and sweat rates calibrated by transferring the sweat sample into a constant bore calibration pipette. The typical time course of sweating response is shown for both $\mathrm{MCH}$ and ISO injection in a normal subject (Fig. 3). Since the sweating rate for ISO was low, sweat was collected every $10 \mathrm{~min}$, and the first 10 min sweat rate was used as the maximal ISO sweat rate, whereas the $\mathrm{MCH}$ sweat rate was determined every $5 \mathrm{~min}$ and the maximal sweat rate usually achieved between a 5 - and 15 -min period. The maximal number of active sweat pores inside the chamber (range 43-113/0.38 $\mathrm{cm}^{2}$ ) were counted during stimulation with $\mathrm{MCH}$, which was then used to calculate the sweat rate per gland per min. The same maximal pore number during $\mathrm{MCH}$ stimulation was used to calculate the ISO-induced sweat rate although the number of active pores tended to be lower during stimulation with ISO with or without TH.

Concentrations of drugs in injection solutions. To circumvent the difficulty in performing a dose-response study in vivo (see Discussion), we used the maximal sweat rate as a practical measure of pharmacological sensitivity and the overall glandular activity of the sweat gland (9).

In preliminary experiments in three control subjects (i.e., a poor sweater, an intermediate sweater, and a heavy sweater, by history), we

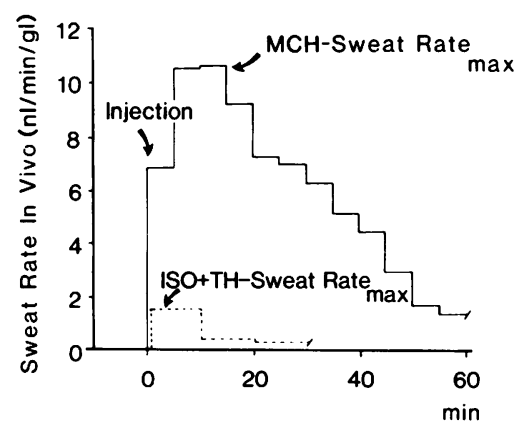

Figure 3. An illustrative example of the time course of sweating response to intradermal injection of $\mathrm{MCH}(5$ $\times 10^{-4} \mathrm{M}$ ), and a combination of ISO $(8$ $\left.\times 10^{-5} \mathrm{M}\right)$ and $\mathrm{TH}$ $\left(10^{-2} \mathrm{M}\right)$ in a normal control. Drugs were injected at time zero. 
injected from $2.5 \times 10^{-3}$ to $2 \times 10^{-5} \mathrm{M} \mathrm{MCH}$ and followed the time course of sweat response using the modified anaerobic method described earlier. In all three subjects, we found that $5 \times 10^{-4} \mathrm{M} \mathrm{MCH}$ concentration consistently gave the highest sweat rate, and the higher $\mathrm{MCH}$ concentration usually produced comparable or even a lesser sweat rate. Thus, $5 \times 10^{-4} \mathrm{M} \mathrm{MCH}$ was used throughout the study.

Concentrations of other drugs used for intradermal injection are listed in Table I. AT $\left(1.4 \times 10^{-4} \mathrm{M}\right)$ was always injected with adrenergic drugs (i.e., ISO, EP, phenylephrine [PL]) to inhibit any possible cholinergic component of sweating. A few CF patients did show transient sweating when ISO + TH without AT was injected intradermally. However, this response was totally inhibited by $1.4 \times 10^{-4} \mathrm{M} \mathrm{AT}$. Injection of as high as $10^{-2} \mathrm{M}$ AT does not influence beta adrenergic responsiveness $(n=6)$. $10 \mathrm{mM}$ TH included for ISO injection was prepared by adding $5 \mathrm{mM}$ aminophylline (dimer of theophylline bound to ethylenediamine, U.S. Pharmacopeia grade). Injection of $10 \mathrm{mM}$ TH alone failed to cause sweating in control subjects (not illustrated). That TH + ISO injection indeed reflects amplified beta adrenergic stimulation is best shown in Fig. 4, where propranolol (PR, obtained as Inderal from Ayerst, Inc., New York) inhibited ISO + TH-induced sweating in a dose-related manner. PR $\left(10^{-5}\right.$ to $\left.5 \times 10^{-4} \mathrm{M}\right)$ has no effect on $\mathrm{MCH}$ sweating in vitro (5) and in vivo (not shown). Further justification for the use of TH will be discussed in later sections. ISO, EP, PL, and AT were conveniently prepared fresh from injection ampoules (U. S. Pharmacopeia grade) and the $\mathrm{MCH}$ solution was prepared at the University of Iowa Hospitals pharmacy by dissolving MCH powder (purchased from Sigma Chemical Co.) in Ringer's solution.

Determination of tissue cAMP level. The methods for incubation of the sweat glands, extraction and reconstitution of cAMP, and determination of CAMP by radioimmunoassay have been described previously

Table I. Approximate Concentrations of Drugs Used for Intradermal Injection

\begin{tabular}{|c|c|c|}
\hline & Injection solutions & \\
\hline ISO: & $\begin{array}{l}\text { Isoproterenol, } \\
\text { Atropine }\end{array}$ & $\begin{array}{l}2.4 \text { or } 8 \times 10^{-5} \mathrm{M} \\
1.4 \times 10^{-4} \mathrm{M}\end{array}$ \\
\hline \multirow[t]{2}{*}{ ISO + TH: } & $\begin{array}{l}\text { Isoproterenol, } \\
\text { Theophylline } \\
\text { (Aminophylline) }\end{array}$ & $\begin{array}{l}8 \times 10^{-5} \mathrm{M} \\
10^{-2} \mathrm{M}\end{array}$ \\
\hline & Atropine & $1.4 \times 10^{-4} \mathrm{M}$ \\
\hline \multirow[t]{2}{*}{ PL: } & $\begin{array}{l}\text { Phenylephrine } \\
\text { (Neosynephrine) }\end{array}$ & $5 \times 10^{-3} \mathrm{M}$ \\
\hline & $\begin{array}{l}\text { Atropine } \\
\text { Propranolol }\end{array}$ & $\begin{array}{r}1.4 \times 10^{-4} \mathrm{M} \\
5 \times 10^{-4} \mathrm{M}\end{array}$ \\
\hline EP: & $\begin{array}{l}\text { Epinephrine, } \\
\text { Atropine }\end{array}$ & $\begin{array}{r}5 \times 10^{-4} \mathrm{M} \\
1.4 \times 10^{-4} \mathrm{M}\end{array}$ \\
\hline MCH: & Methacholine & $5 \times 10^{-4} \mathrm{M}$ \\
\hline
\end{tabular}

These solutions were conveniently prepared fresh from injection ampoules and diluted to final concentration by adding Ringer's solution prepared for intravenous infusion. $0.2 \mathrm{ml}$ of injection solution was used in order to cover the test site of $0.38 \mathrm{~cm}^{2}$ with blanching injection wheal.

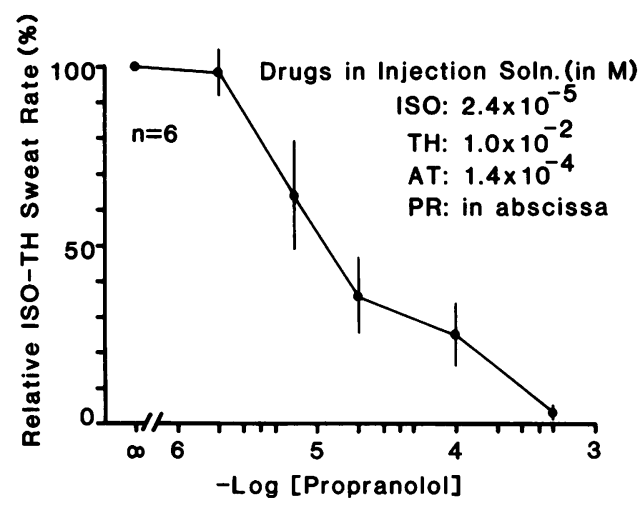

Figure 4. Effect of PR on ISO + TH-induced sweating. Each plot is the mean \pm SEM of six subjects.

$(6,10)$. The number of sweat glands isolated from each biopsy specimen varied but usually $40-80$ sweat glands were available for CAMP assay. Since the size of the sweat gland differs, especially among different individuals and to a certain extent even in the same biopsy specimen, tissue cAMP levels were expressed on the per unit protein basis, i.e., picomoles per microgram protein. Typical experimental protocols are as follows.

2 or 3 sweat glands were preincubated for $10 \mathrm{~min}$ at $37^{\circ} \mathrm{C}$ in each test tube containing $100 \mu \mathrm{l} \mathrm{KRB}$ without albumin (albumin is not essential for sweat induction nor for CAMP production, unpublished observation), and at time zero, drugs were added to the medium. At the end of each incubation period, sweat glands were picked up as a single mass with a pair of sharp forceps under microscopic control, snap frozen in liquid nitrogen, and then transferred to an Eppendorf (Brinkmann Instruments Inc., New York) micro test tube with an attached cap containing $50 \mu \mathrm{l}$ of $0.1 \mathrm{~N} \mathrm{HCl}$. The sweat glands in the $\mathrm{HCl}$ solution were then boiled for $5 \mathrm{~min}$ at $100^{\circ} \mathrm{C}$ in order to extract cAMP from the tissue. An aliquot of the $\mathrm{HCl}$ solution (extracting cAMP) was then lyophilized and the cAMP solution reconstituted by adding $20 \mu \mathrm{l}$ of $0.05 \mathrm{M}$ acetate buffer (pH 6.2). An aliquot of the reconstituted buffer was subsequently subjected to acetylation and radioimmunoassay according to the New England Nuclear (Boston, MA) kit. The identity of the nucleotide detected by RIA as cAMP has been elaborated in a previous communication (10). Protein content in each test tube was determined by measuring the Lowry protein content of the incubated sweat glands recovered from the $\mathrm{HCl}$ solution (extracting cAMP) under stereomicroscopic control and soluble protein concentration in the $\mathrm{HCl}$ solution and the original KRB incubation medium. The protein content of CF sweat glands was $2.54 \pm 0.34$ (mean \pm SEM) and that of control was $3.12 \pm 0.48 \mu \mathrm{g} /$ gland. Extracellular cAMP released into the incubation medium during incubation was less than $5 \%$ of cAMP accumulated in the sweat gland. Since further dissection of the whole human sweat gland into the secretory portion and the ductal portion is extremely tedious and since stimulated tissue cAMP level is nearly totally due to the secretory portion (10), no further attempt was made in this study to measure cAMP in both the secretory portion and the duct.

In preliminary experiments, recovery of tissue cAMP was compared between control and CF sweat glands. First, tissue cAMP was repeatedly extracted by $0.1 \mathrm{~N} \mathrm{HCl}$ at $100^{\circ} \mathrm{C}$ by transferring the sweat glands to new test tubes after the first extraction. In both control and CF glands, the cAMP content in the second extractants was negligible. Second, in 
order to estimate the extent of loss of cAMP by adsorption to the tissue, enzymatic degradation by the tissue, or by inefficiency of cAMP extraction, 1-3 pmol of exogenous cAMP were added to both CF and control sweat glands incubated with AT and PR and to appropriate tissue blanks before subjection to cAMP extractions. The estimated loss of exogenous cAMP was $<10 \%$ in both control and CF glands.

TH was used throughout the study as a phosphodiesterase inhibitor because in our previous study (6) TH was comparable with MIX (1methyl-3-isobutylxanthine) in vitro. Furthermore, MIX requires the use of organic solvents which could alter cAMP metabolism (11) and because the in vitro study could be compared with the in vivo injection study. Unless otherwise stated, all the reagents were obtained from Sigma Chemical Co.

\section{Results}

Sweat induction in vitro. Abnormal responsiveness of isolated CF sweat glands was discovered when we started dissecting CF sweat glands and studying their pharmacological responsiveness in vitro in 1980. Fig. 5 illustrates an example of such experiments. After isolating the sweat gland and holding it in a constriction pipette (see Fig. 1), the sweat gland was stimulated first with 3 $\times 10^{-6} \mathrm{M}$ and then $5 \times 10^{-5} \mathrm{M} \mathrm{MCH}$, both of which induced copious sweat secretion. Then, at "wash," the incubation medium was replaced several times with fresh medium containing $10^{-7}$ M AT, which completely stopped sweating. As can be seen, subsequent stimulation with varying concentrations of ISO, even with maximal potentiation with $10^{-2} \mathrm{M} \mathrm{TH}$, failed to induce the secretory response. The lack of secretory response to ISO + AT may not be due to inhibition of glandular function by ISO + TH because addition of $5 \times 10^{-5} \mathrm{M} \mathrm{MCH}$ at $70 \mathrm{~min}$ induced a maximal response that was then inhibited by $10^{-6}$ $\mathrm{MAT}$ at $90 \mathrm{~min}$. In contrast, sweat glands from normal controls responded to varying extents to ISO alone (Fig. $6 A$ ) or to ISO + TH (Fig. $6 B$ and $C$ ).

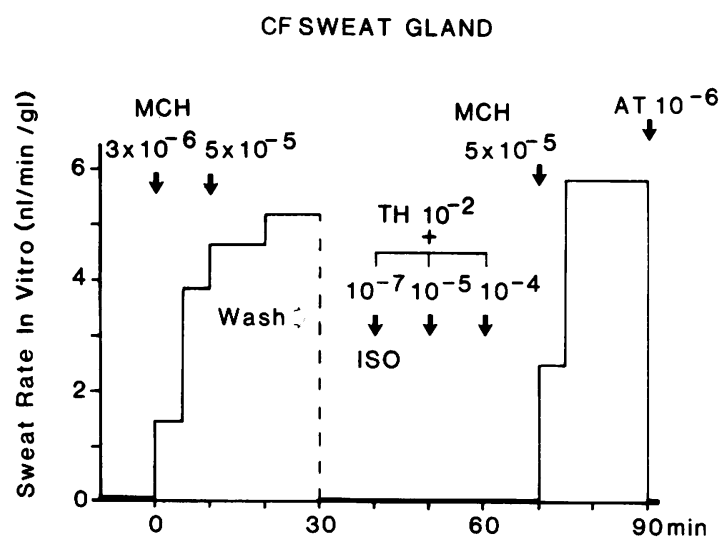

Figure 5. An illustrative in vitro experiment showing the lack of responsiveness by the CF sweat gland to ISO + TH added at $40 \mathrm{~min}$. Although $10^{-3} \mathrm{M}$ TH was usually used to potentiate the effect of ISO, $10^{-2} \mathrm{M}$ was used on this gland to achieve the maximal potentiation. All the concentrations are given in molar.

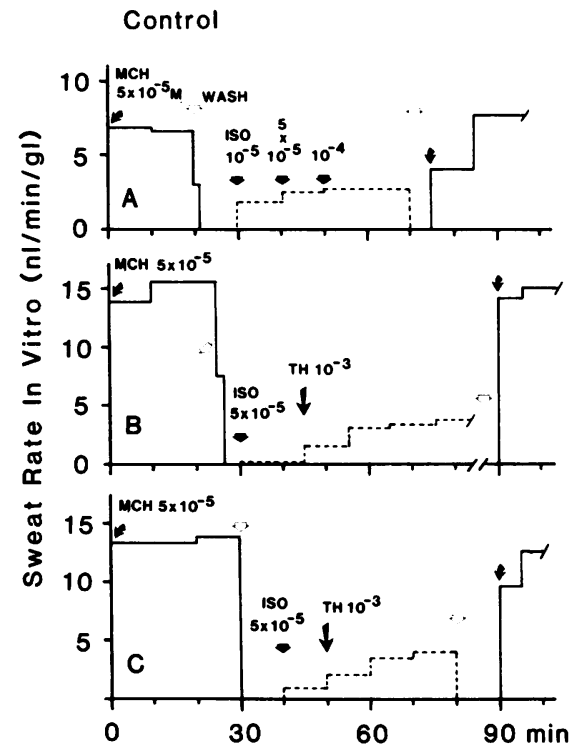

Figure 6. Illustrative experiments showing three sweat glands from normal control. The experimental protocol is similar to that in Fig. 5. The sweat gland in $A$ responded avidly to ISO alone (without $\mathrm{TH}$ ), whereas the gland in $B$ was activated only when TH was added to ISO. ISO-induced sweating in $C$ was markedly augmented by subsequent $\mathrm{TH}$. The long solid curved arrows indicate $\mathrm{MCH}$ addition; empty short arrows denote wash. As in Fig. 5, at "wash" the medium was replaced with fresh KRB containing $10^{-6} \mathrm{M}$ AT.

To further elaborate the interaction between ISO and TH, the effect of $10^{-3} \mathrm{M}$ TH was studied on ISO-induced sweating in 9 control and $12 \mathrm{CF}$ sweat glands (Fig. 7). None of the $\mathrm{CF}$ sweat glands responded to ISO alone or to the combination of ISO and TH, whereas in the control sweat glands, TH markedly augmented ISO-induced sweating in all but one gland.

Fig. 8 summarizes the secretory rate of all the isolated human sweat glands, some of which have already been included in Fig. 7. As can be seen, the $\mathrm{MCH}$ sweat rate max $_{\max }$ of $\mathrm{CF}$ glands is about two-thirds that of control, i.e., $7.3 \pm 0.75$ vs. $4.9 \pm 0.76$. This could be partially explained by the smaller size of CF glands (note

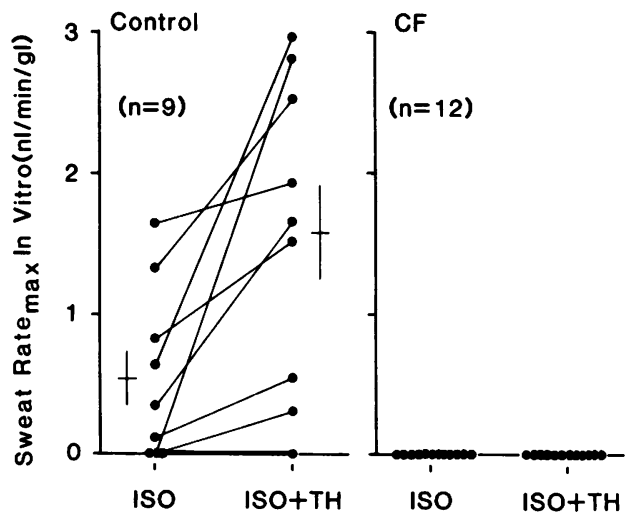

Figure 7. Effect of TH on ISO-induced sweating in vitro. Each line indicates an experiment from a single gland. Sweat glands were obtained from 11 control subjects and $8 \mathrm{CF}$ patients. The horizontal bars are the mean, and the vertical bars \pm SEM. $n$, no. of sweat glands studied. 


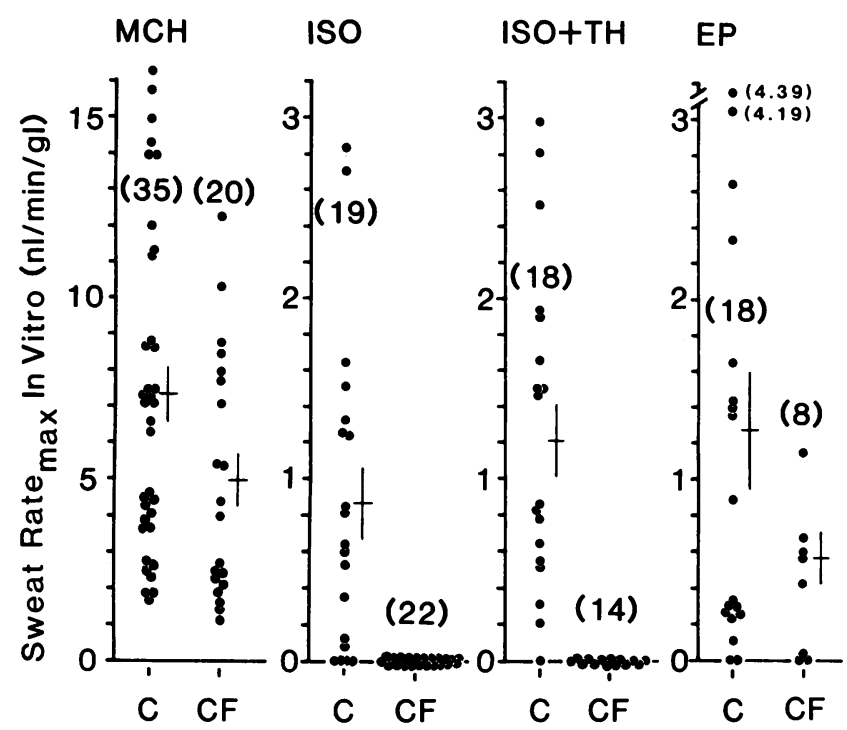

Figure 8. Summary of sweat rate $\max _{\max }$ of $\mathrm{CF}$ and control sweat glands under various pharmacological stimulation in vitro. Concentrations of the drugs used are: $\mathrm{MCH}, 5 \times 10^{-5} \mathrm{M}$; ISO, $5 \times 10^{-5} \mathrm{M}$; TH, $10^{-3} \mathrm{M}$; EP, $5 \times 10^{-5} \mathrm{M}$. The crosses are the means \pm SEM. C, control glands; $\mathrm{CF}$, cystic fibrosis glands. The numbers in parentheses in each column are the number of glands studied. Small numbers in parentheses indicate the actual sweat rate of the plots located off the scale.

the average protein content of CF glands was only $77 \%$ of control). Fig. 8 also suggests that responsiveness of $\mathrm{CF}$ glands to alpha adrenergic stimulation may be normal because EP (a stimulant of both alpha and beta adrenergic receptors) induced sweating in 5 of $8 \mathrm{CF}$ glands with a wide overlap with control glands.

Intradermal sweat test in vivo. Prompted by these foregoing preliminary in vitro observations, we asked whether the lack of beta adrenergic sweat secretion of the CF sweat gland can be reproduced also in vivo. Drug concentrations used for intradermal injection were in general ten times those used in vitro because injected drugs will be diluted in the skin manyfold (also see Methods). The importance of using TH for potentiating beta stimulation is best illustrated in Fig. 9. At first, we presumed that the higher concentrations of ISO might still achieve the maximal beta adrenergic sweat rate without the use of TH. However, the highest ISO concentration, $8 \times 10^{-4} \mathrm{M}$, did not necessarily yield a sweat rate greater than that achieved by $8 \times 10^{-5} \mathrm{M}$ (not shown). Moreover, some subjects complained of palpitation after intradermal injection of $0.2 \mathrm{ml} 8 \times 10^{-4} \mathrm{M}$ ISO, suggesting systemic absorption and thus precluding the use of this concentration. Thus, for the following intradermal injection study, unless otherwise noted, we used $8 \times 10^{-5} \mathrm{M}$ ISO with $10^{-2} \mathrm{M}$ TH in order to induce the maximal beta adrenergic sweating. It is to be noted that none of the CF patients responded to intradermal ISO; however, about half of the controls also failed to respond. With $10^{-2} \mathrm{M}$ TH (with $1.4 \times 10^{-4}$ M AT as a necessary cholinergic inhibitor) added to ISO, the sweat rate increased markedly in all the control subjects so that even those control subjects who failed to respond to ISO alone had now responded to the combination of ISO and TH (Fig. 9). Fig. 10 is the summary of the maximal sweat rate due to intradermal ISO $+\mathrm{TH}$ in all the subjects. Note the striking similarity to the in vitro results shown in Fig. 8. Also, note the lower than control ISO + TH sweat rate in CF heterozygotes, although the overlap with control is not ignorable (Fig. 10). Although the mean $\mathrm{MCH}$ sweat rate max $_{\text {in }}$ the $\mathrm{CF}$ patients was slightly lower than in the controls $(7.9 \pm 0.66$ vs. $5.7 \pm 0.60)$, the wide overlap between the two groups and the extent of individual variation in the controls complicate its interpretation. The responsiveness of the CF patients to the predominantly alpha adrenergic agent, PL, is also comparable with that of the controls, indicating that the alpha adrenergic responsiveness of $\mathrm{CF}$ sweat glands is apparently normal.

Tissue cAMP accumulation in isolated human sweat glands. Fig. 11 illustrates the dose response to ISO of tissue cAMP accumulation in isolated sweat glands from CF patients and age-matched controls. As in isolated monkey palm sweat glands $(6,10)$, the tissue cAMP level is a saturable function of ISO concentration, plateauing at about $5 \times 10^{-5} \mathrm{M}$. At no concentrations was the difference between $\mathrm{CF}$ and control significant $(P>0.05)$. Fig. 12 compares the time course of tissue cAMP accumulation during stimulation with ISO and ISO + TH between CF and control glands. Since the protein content and thus the size of the sweat gland differs among different individuals (9) and between control and CF, cAMP level was related to protein content in the sweat gland. When cAMP level is expressed on the per gland basis, CF glands showed the lower cAMP level (not shown). However, when expressed on the per microgram protein basis (Fig. 12), nonstimulated and ISO-stimulated cAMP levels were not significantly different between the two groups

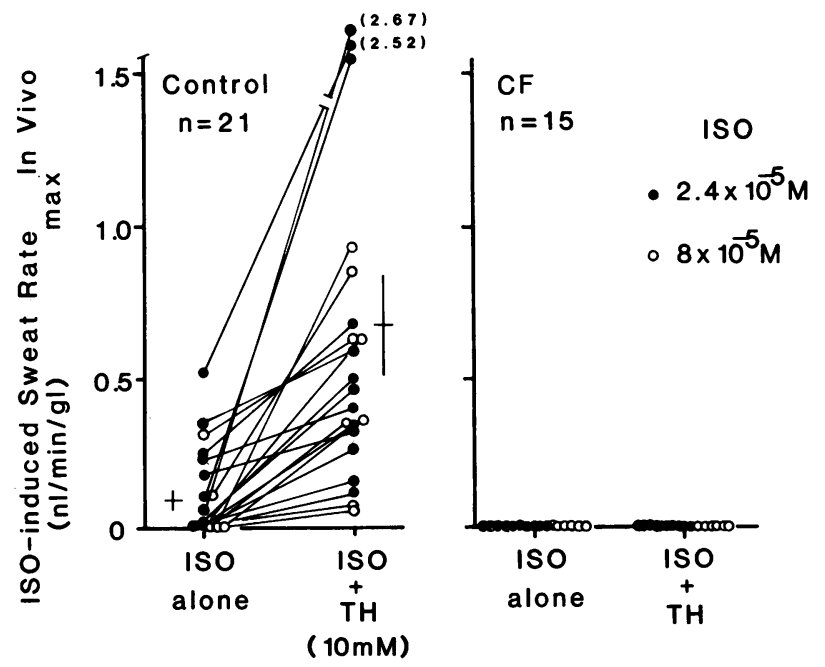

Figure 9. Effect of TH on ISO-induced sweating in vivo. In each subject, either or both of 2.4 and $8 \times 10^{-5} \mathrm{M}$ ISO with or without TH were injected intradermally (i.e., one or two experiments were derived from each subject). $n$, no. of experiments. 


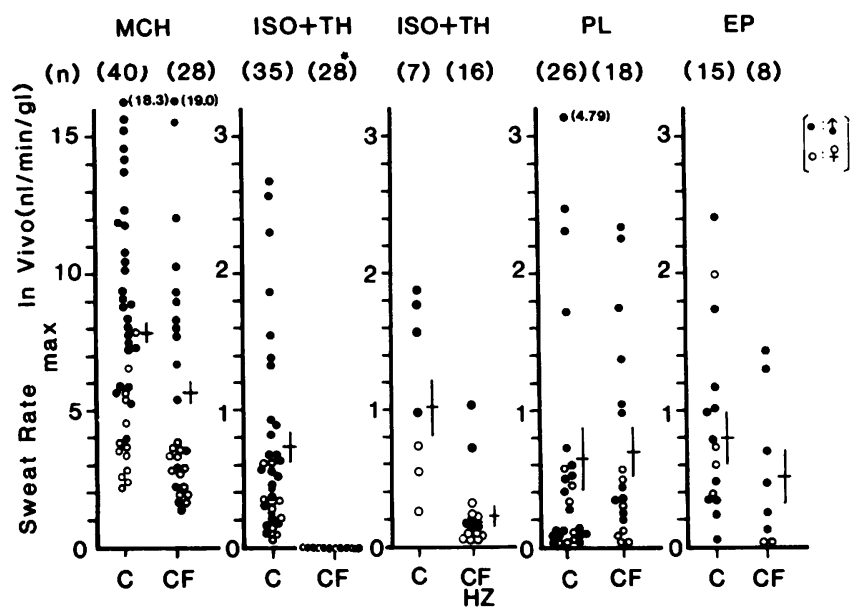

Figure 10. Summary of sweat rate $\max _{\max }$ in vivo of $\mathrm{CF}$ and control following intradermal injection of various drugs. Concentrations of injected drugs and other necessary inhibitors are listed in Table I. The numbers in large parentheses are the no. of subjects studied. Empty symbols are female and the solid symbols are males. Crosses are the means \pm SEM. 2 of $28 \mathrm{CF}$ patients showed a transient uncollectable amount of sweat secretion (sweat rate $<0.007 \mathrm{nl} / \mathrm{gl}$ per min) during stimulation with ISO + TH. The repeated sweat test, however, failed to reproduce this minute ISO-sweating. Asterisk, the number of $\mathrm{CF}$ subjects should read 24 if it could later be proven that adrenergic agonists taken by 4 of $28 \mathrm{CF}$ on an infrequent basis during the preceding $2 \mathrm{mo}$ had influenced their sweating response to intradermal ISO (also see footnote 4); CFHZ, cystic fibrosis heterozygotes compared with their age- and sex-matched controls.

( $P \geq 0.05$ ), nor was the peak level of cAMP level stimulated by both ISO and TH, i.e., at $10 \mathrm{~min}$. The only difference between the two groups is the slower rate of rise of cAMP level in the CF sweat glands when stimulated with both ISO and TH during the first $5 \mathrm{~min}$ of incubation (i.e., plots $a^{\prime}$ and $b^{\prime}$ in Fig. 12). The contribution of contaminating periglandular components,

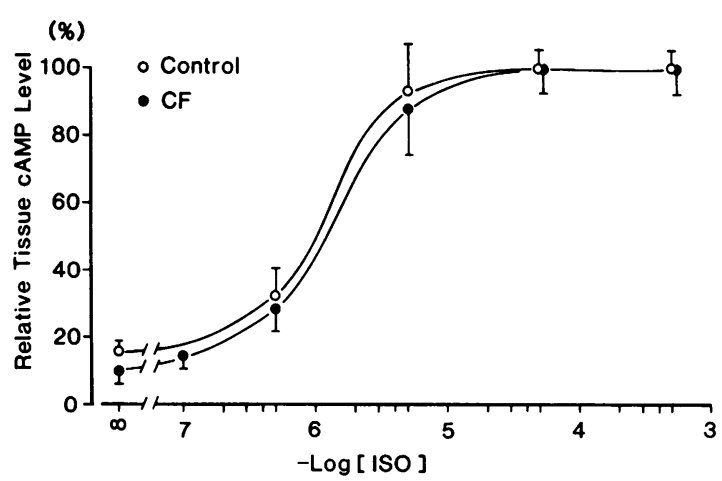

Figure 11. Dose response to ISO of tissue cAMP accumulation. Each plot is the mean \pm SEM of from 6 to 16 determinations in six subjects. To minimize differences in cAMP levels in different individuals, cAMP levels were expressed as percentile of the maximal level in each subject. All the sweat glands were incubated for $10 \mathrm{~min}$ with ISO. $P>0.05$ for all pairs of corresponding plots.

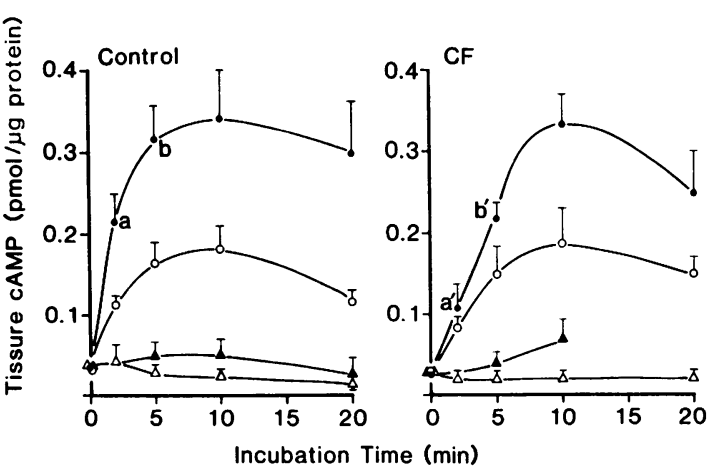

Figure 12. Time course of tissue cAMP accumulation of isolated human sweat glands. cAMP level is expressed on the per microgram protein basis. The effect of TH was studied on both basal cAMP level (AT and PR $[\Delta]$ vs. AT and PR and TH [ $\Delta]$ ) and ISO-stimulated cAMP level (ISO and AT [O] vs. ISO and AT and TH [•]). Concentrations of the drugs used are: AT, $5 \times 10^{-6} \mathrm{M} ; \mathrm{PR}, 5 \times 10^{-6} \mathrm{M}$; $\mathrm{TH}, 10^{-3} \mathrm{M}$; ISO, $5 \times 10^{-5} \mathrm{M}$. Each plot is the mean \pm SEM of from 6 to 16 determinations using sweat glands obtained from $6 \mathrm{CF}$ and 6 control subjects. $P<0.001$ between $a$ and $a^{\prime}$ and $b$ and $b^{\prime} . P$ values between all other corresponding plots (i.e., control vs. CF) are $>0.05$. AT and PR were added as indicated as necessary inhibitors.

if any, to the cAMP level may be negligible because incubation of periglandular connective tissues comparable in size to those usually attached to the sweat gland $(\sim 10 \%$ of the protein content in the whole sweat gland) showed a negligible amount of cAMP accumulation.

\section{Discussion}

We observed that, unlike control sweat glands, CF glands fail to show a secretory response to a beta adrenergic agonist, ISO, both in vivo and in vitro. The pharmacological responsiveness of isolated monkey palm eccrine sweat glands to cholinergic alpha and beta adrenergic stimulation has been elaborated previously in the authors' laboratory (5). Although qualitative pharmacological studies of the human eccrine sweat gland in vivo have been documented repeatedly over the past four decades $(12,13)$, any attempts at conducting more precise quantitative pharmacological studies of human sweat glands have met numerous technical barriers. First, in in vivo experiments, periglandular concentrations of drugs introduced into the skin either by iontophoresis or intradermal injection are never known because introduced drugs are immediately diluted by diffusion and by periglandular blood circulation. Second, a wide individual variation exists in cholinergic responsiveness, anatomical dimensions, and the secretory activity of the gland (9), so that parameters of pharmacological responsiveness (as expressed by $\mathrm{p} K_{\mathrm{A}}$ 's, affinity constants, for various agonists and $\mathrm{p} K_{\mathrm{B}}$ 's for various blockers) are applicable only to that particular individual at the time the test was conducted. Third, as compared with the monkey palm eccrine sweat glands, isolation without damage of human eccrine sweat glands for sweat induction study in 
vitro is far more difficult and time-consuming. We have previously suggested (9) that one possible solution to this dilemma is to use the maximal sweat rate per gland as a measure of overall glandular activities because the higher the sweat rate max $_{\max }$ per gland or per unit tubular length of the secretory coil, the higher is the pharmacological sensitivity (as defined by the higher affinity constant for $\mathrm{MCH}, \mathrm{p} K_{\mathrm{A}}$ ) and the larger is the size of the sweat gland. Since $\mathrm{p} K_{\mathrm{A}}$ for $\mathrm{MCH}$ and perhaps also for ISO differ in different individuals, the only way to insure obtaining the maximal sweat rate from different subjects is to use the supramaximal concentrations for in vivo (see Table I) and in vitro experiments as done in this study.

An erratic or irreproducible sweating response to intradermal injection of adrenergic drugs has long been known (14). Although this has been attributed to vasoconstriction caused by (nor)epinephrine or by PL, the erratic response to intradermal injection of ISO (which does not cause vasoconstriction) has been a puzzle. ${ }^{2}$ We found that addition of TH to ISO markedly augments beta responsiveness of normal sweat glands both in vivo and in vitro. This supports the generally held theory that beta adrenergic stimulation is mediated by intracellular cAMP levels $(6,10,15)$, and that TH augments the effect of ISO principally by minimizing phosphodiesterase-induced degradation of intracellular cAMP. This interpretation appears also to be true with the sweat gland because $(a) \mathrm{TH}$ alone is not sudorific in vivo or in vitro in the human (unpublished data), (b) TH plus ISO-induced sweating is inhibited by PR in a dose-dependent fashion, and (c) TH indeed augmented ISO-induced cAMP accumulation in isolated eccrine sweat glands.

The present study has shown a distinct separation between CF patients ${ }^{3}$ and their age and sex-matched controls ${ }^{4}$ in terms of their sudorific response to ISO plus TH both in vivo and in vitro. No CF sweat glands responded to augmented beta adrenergic stimulation, whereas all but one of the control sweat

2. We would like to reiterate that deep intradermal injection or subcutaneous injection of ISO + TH can cause submaximal sweat rate or even pseudo negative response test in normal subjects. The drug must be injected into the upper dermis (where the sweat glands are located) in such a way as to cause a blanching injection wheal (or an orange peel look of the skin).

3. Using an improved iodine-starch paper technique, we have been able to confirm the present in vivo finding on defective beta adrenergic sweating in infants and young children with CF (16).

4. The possibility that defective beta adrenergic responsiveness of CF patients could be due to acquired desensitization of beta receptors by therapeutic ingestion of adrenergic drugs appears unlikely because the majority of CF patients did not take adrenergic agonists (see Methods) and because three adult patients with severe asthma and one patient with severe asthma and atopy who had continuously taken TH plus beta agonists responded normally to intradermal injection of isoproterenol as well as to MCH. (Age 20-23; two males and two females. MCH sweat rate $_{\max }, 2.99 \pm 0.94 \mathrm{nl} / \mathrm{min}$ per $\mathrm{gl}$ ISO + TH sweat rate, $0.36 \pm 0.10 \mathrm{nl} /$ min per gl; relative ISO + TH sweat rate, $12 \%$.) One patient with generalized atopic dermatitis without adrenergic agonists responded to both MCH (sweat rate $\max , 3.0 \mathrm{nl} / \mathrm{min}$ per gland) and ISO + TH $(0.39 \mathrm{nl} /$ min per gland). glands did to varying extents. In contrast, the response of $\mathrm{CF}$ sweat glands to $\mathrm{MCH}$, EP (alpha and beta adrenergic agonist) and PL (alpha agonist) were comparable with control. These observations indicate that it is only the beta adrenergic component that may be abnormal in the CF sweat gland.

We then made a limited attempt at distinguishing the site of defect in CF, such as beta receptor-agonist interaction, adenylate cyclase, or (increased) phosphodiesterase activity. Contrary to our initial expectation, isolated and incubated CF sweat glands showed normal tissue accumulation of cAMP in response to varying doses of ISO. Furthermore, the maximal tissue cAMP level in CF glands (by ISO + TH at 10 min of incubation in Fig. 12) was also comparable with control although the time course of ISO + TH-induced cAMP accumulation during the first $5 \mathrm{~min}$ of incubation was significantly lower than in controls. It is not certain whether this latter observation is relevant to the defective beta adrenergic-induced sweating in CF; however, we are somewhat skeptical of such a possibility because in the in vitro sweat induction study, the CF sweat gland does not respond to ISO + TH at all, even if exposed to these drugs for a prolonged period of time, i.e., for as long as $\mathbf{3 0 ~ m i n . ~ A l t h o u g h ~}$ the observed CAMP accumulation may largely reflect that in the secretory coil, ${ }^{5}$ the critical question remains unanswered as to whether the secretory cells (rather than dark cells or myoepithelial cells) are predominantly responsible for cAMP accumulation in both control and CF. Nevertheless, in a recessive disease where an abnormal gene product may presumably be involved regardless of cell types, the measurement of cAMP response in the whole tissue itself may serve an initial screening purpose. Thus, until more refined information becomes available, the present cAMP study should be regarded as tentative in nature.

The present observation has also raised another important issue, that is, how is this defect of the CF secretory coil related to the defective ductal $\mathrm{NaCl}$ reabsorption by the $\mathrm{CF}$ sweat duct? No speculation on this can be made at this point. An abnormality of autonomic nervous function has frequently been suggested as the possible etiology of CF. Evidence for this possibility includes: chronically reserpinized rats resemble $\mathrm{CF}$ in many respects $(17,18)$; isoproterenol-treated rat was once proposed as a model system for CF (19); decreased effect of ISO on the heart rate but an increased sensitivity to PL on pupillary dilation is

5. The whole sweat gland used in this study consists of the duct which occupies $\sim 1 / 3-1 / 4$ of total protein content and the secretory coil (unpublished data). In the monkey sweat glands, an ideal model for human glands both structurally and functionally, both the duct and the secretory coil accumulate cAMP to the same extent when expressed on a per unit protein basis, with and without ISO stimulation (10). Thus, unless the CF sweat duct produces three- to fourfold more cAMP accumulation (a less likely possibility because the duct is not hypertrophied in CF), the observed cAMP level in Figs. 11 and 12 should reflect largely that in the secretory coil although this presumption must be confirmed in the future. Furthermore, the present presumption that the secretory cells account in large part for cAMP level in the secretory coil must also be confirmed. 
reported in CF (20); and ISO-induced cAMP production is decreased (21) and beta adrenergic receptor binding is impaired (22) in CF leukocytes, but a more recent paper (23) reports normal beta adrenergic receptor binding and cAMP response in CF granulocytes. Thus, the present study may complement other related observations in the overall search for the basic defect for this semilethal genetic disease of young children.

\section{Acknowledgments}

Thanks are due to Ms. M. Washburn for help in preparation of the manuscript, to Ms. M. J. Boeding for technical help in the in vivo sweat test, to Dr. M. Weinburger for generously allowing access to the list of CF patients in the Cystic Fibrosis Center at the University of Iowa, and to Dr. M. Welsh for critically reading the manuscript.

This work was supported in part by U. S. Public Health Service grants nos. AM27857 and AM25339.

\section{References}

1. Di Sant'Agnese, P. A., and P. B. Davis. 1976. Research in cystic fibrosis. N. Engl. J. Med. 295:481-485, 534-541, 597-602.

2. Schulz, I. J. 1969. Micropuncture studies of the sweat formation in cystic fibrosis patients. J. Clin. Invest. 48:1470-1477.

3. Mangos, J. A. 1973. Microperfusion study of the sweat gland abnormality in cystic fibrosis. Tex. Rep. Biol. Med. 31:651-663.

4. Sato, K. 1982. Mechanism of Eccrine Sweat Secretion. In Fluid and Electrolyte Abnormalities in Exocrine Glands in Cystic Fibrosis. P. M. Quinton, J. R. Martinez, and U. Hopfer, editors. San Francisco Press, San Francisco, CA. 35-52.

5. Sato, K., and F. Sato. 1981. Pharmacologic responsiveness of isolated single eccrine sweat glands. Am. J. Physiol. 240:R44-R51.

6. Sato, K., and F. Sato. 1981. The role of cyclic AMP in the beta adrenergic mechanisms of sweat secretion. Pfleugers Arch. Eur. J. Physiol. 390:49-53.

7. Sato, K., and F. Sato. 1981. Role of calcium in cholinergic and adrenergic mechanisms of eccrine sweat secretion. Am. J. Physiol. 241:C113-C120.

8. Brusilow, S. W. 1965. An anaerobic sweat collection technique. J. Lab. Clin. Med. 65:513-517.

9. Sato, K., and F. Sato. 1983. Individual variations in structure and function of human eccrine sweat gland. Am. J. Physiol. 245(Regulatory, Integrative, Comp. Physiol. 14):R203-R208.

10. Sato, K., and F. Sato. 1983. Cholinergic potentiation of isoproterenol-induced cyclic AMP level in the sweat gland. Am. J. Physiol. 245(Cell Physiol. 14):C189-C195.

11. Harper, J. F., and G. Brooker. 1980. Alcohol potentiation of isoproterenol-stimulated cyclic AMP accumulation in rat parotid. $J$. Cyclic Nucleotide Res. 6:51-62.

12. Randal, W. C., and K. K. Kimura. 1955. The pharmacology of sweating. Pharmacol. Rev. 7:365-397.

13. Sato, K. 1977. The physiology, pharmacology and biochemistry of the eccrine sweat gland. Rev. Physiol. Biochem. Pharmacol. 79:51131.

14. Rothman, S. 1954. Physiology and Biochemistry of the Skin. The University of Chicago Press, Chicago. 167.

15. Robison, G. A., R. W. Butcher, and E. W. Sutherland. 1971. Cyclic AMP. Academic Press, New York. 419.

16. Boote, W., M. Weinburger, and K. Sato. 1983. Sweat patch test as a sensitive screening test for diagnosis of cystic fibrosis. Clin. Res. 31:797A.

17. Martinez, J. R., E. Adelstein, D. Quissel, and G. J. Barbero. 1975. The chronically reserpinized rat as a possible model for cystic fibrosis. I. Submaxillary gland morphology and ultrastructure. Pediatr. Res. 9:463-469.

18. Martinez, J. R., A. M. Martinez, L. Garrett, and P. Korman. 1979. Chronically reserpinized rat as a model for cystic fibrosis: $\mathrm{Na}$ transport inhibitory effect in submaxillary saliva. Pediatr. Res. 13:11561159.

19. Mangos, J. A., N. R. McSherry, and P. J. Benke. 1969. Studies on the pathogenesis of cystic fibrosis: the isoproterenol treated rat as an experimental model. Proc. Fifth Int. Cystic Fibrosis Conf. D. Lawson, editor. Cystic Fibrosis Research Trust, Cambridge, England. 25-34.

20. Davis, P. B., J. R. Shelhamer, and M. Kaliner. 1980. Abnormal adrenergic and cholinergic sensitivity in cystic fibrosis. N. Engl. J. Med. 302:1453-1456.

21. Davis, P. B., M. Braunstein, and C. Jay. 1978. Decreased adenosine $3^{\prime}, 5^{\prime}$-monophosphate response to isoproterenol in cystic fibrosis leucocytes. Pediatr. Res. 12:703-707.

22. Galant, S. P., L. Norton, J. Herbst, and C. Wood. 1981. Impaired beta adrenergic receptor binding and function in cystic fibrosis neutrophils. J. Clin. Invest. 68:253-258.

23. David, B. D., L. Dieckman, T. F. Boat, R. C. Stern, and C. F. Doershuk. 1983. Beta adrenergic receptors in lymphocytes and granulocytes from patients with cystic fibrosis. J. Clin. Invest. 71:1787-1795. 